\title{
Aspects neurobiologiques de la fonction sexuelle masculine
}

\section{Sommaire}

Neurophysiologie centrale de l'érection

344 - Etude des structures cérébrales impliquées dans l'état d'excitation sexuelle S. STOLERU ET AL

345 - Rôle de la dopamine dans le contrôle de l'érection et du comportement sexuel chez le rat E.M. HULL

347 - Rôle du noyau paraventriculaire de l'hypothalamus dans la commande de l'érection A. ARGIOLAS

348 - Régulation médullaire : rôle de l'ocytocine $O$. RAMPIN

Conséquences sexuelles des traumatismes médullaires

350 - Les greffes médullaires de neurones : données expérimentales A. PRIVAT

350 - Le tractus séminal des patients blessés médullaires J.C. SOUFIR

351 - Le recours à l'AMP chez les patients blessés médullaires V. IzARD

353 - Actualité du traitement pharmacologique des troubles de l'érection chez les blessés médullaires $F$. GIULIANO

La sexualité revisitée en 1997 à la lumière de la neurobiologie

356 - Comparaison des fonctions sexuelles chez le mâle et la femelle K.E. MC KENNA

357 - Psychobiologie de l'excitation sexuelle B.D. SACHS

358 - Le point de vue du neuro-endocrinologiste J.D. VINCENT

359 - La sexualité masculine et la chaîne érotique A. GREEN

Réunion conjointe organisée à l'initiative des Comités Interface INSERMREPRODUCTION HUMAINE et INSERM-NEUROSCIENCES, dans le cadre de la Journée d'Andrologie de Bicêtre, 20 juin 1997

Résumés des communications 


\section{Neurophysiologie centrale de l'érection}

\section{Etude des structures cérébrales impliquées dans l'état d'excitation sexuelle}

S. StolerU*, M.C. Gregoire**, J. DeCeTY ${ }^{\circ}$, D. GERARD**, E. LAFARGE**, L. CinOtTI**, F. LAVENNE**, D. LE BARS**, B. MAZOYER ${ }^{\circ}$, F. MAGNIN*, A. SPIRA*, D. COMAR**

*Unité 292 INSERM, Le Kremlin-Bicêtre, 94276 France ; ${ }^{* *}$ CERMEP, Lyon, 69003, France ; 'Unité 94, INSERM, Lyon, 69003, France; ${ }^{\circ}$ Département d'Informatique, Hôpital Robert-Debré, Paris, 75019, France.

Les régions cérébrales impliquées dans le comportement sexuel masculin n'ont pas été caractérisées de manière précise. Le développement actuel des techniques d'imagerie cérébrale fonctionnelle et de l'étude scientifique des émotions et des motivations humaines rend maintenant possible l'étude des bases cérébrales du comportement sexuel humain. Comme pour les autres comportements motivés, le comportement sexuel comprend une phase dite appétitive et une phase consommatoire. C'est sur la première phase, encore appelée "excitation sexuelle", que notre recherche a porté. L'objectif était d'identifier les régions cérébrales impliquées dans l'excitation sexuelle chez des sujets masculins en bonne santé. Nous avons fait l'hypothèse que ces régions étaient représentées par les pôles temporaux, le cortex orbitofrontal et le système limbique.

Sujets et méthodes : 8 sujets masculins volontaires sains furent étudiés à l'aide de la tomographie par émission de positons (TEP). Dans la condition expérimentale, nous avons présenté aux sujets un film sexuellement explicite. Nous avons utilisé deux conditions témoins : 1) dans la première, nous avons présenté aux sujets un film aussi neutre que possible sur le plan émotionnel ; 2) dans la seconde, nous avons présenté un film comique, induisant donc des émotions positives mais non sexuelles; cette deuxième condition témoin fut utilisée pour montrer que les différences potentielles de flux sanguin cérébral régional entre la condition expérimentale et la première condition témoin étaient spécifiquement liées à l'excitation sexuelle et non à une activation émotionnelle positive générale. L'analyse a utilisé la cartographie paramètrique statistique (Statistical Parametric Mapping) pour mettre en évidence les différences de flux sanguin cérébral régional entre les diverses conditions.

Résultats : par rapport à la condition non émotionnelle, lors de la condition avec film sexuellement explicite, il y avait une activation bilatérale du gyrus temporal inférieur, de la tête du noyau caudé droit, de la partie antérieure du gyrus cingulaire gauche, et du gyrus frontal inférieur droit; inversement, aucune région ne fut significativement activée dans la condition neutre comparée à la condition avec film sexuel. De plus, l'activation de certaines des régions citées ci-dessus était positivement corrélée avec le niveau de la testostérone plasmatique. Dans la condition humour comparée avec la condition neutre, les gyri temporaux moyens droit et gauche furent activés ; à l'inverse, le gyrus cingulaire droit, le gyrus orbitofrontal droit, et le gyrus frontal moyen gauche étaient plus activés dans la condition neutre que dans la condition humour. Par comparaison avec la condition humour, dans la condition sexuelle il y avait une activation significative du gyrus cingulaire gauche, du gyrus frontal inférieur droit et du noyau caudé droit. Enfin, par comparaison avec la condition sexuelle, dans la condition humour il y avait une activation significative des gyri temporaux moyens droit et gauche, du gyrus frontal inférieur gauche, du précuneus gauche, et du gyrus frontalis medialis gauche. 
Conclusion : ces résultats mettent en évidence que, chez l'homme, l'excitation sexuelle provoquée par des stimuli visuels est caractérisée par un pattern d'activation à trois volets : (i) une activation temporale inférieure bilatérale, dans une région correspondant aux aires associatives visuelles; (ii) une activation d'une série de régions appartenant au cortex paralimbique (insula, pôle temporal, gyrus cingulaire) ; or, on sait que le cortex paralimbique pourrait jouer un rôle dans la coloration émotionnelle et hédonique de l'expérience vécue; de plus, la partie antérieure du gyrus cingulaire contrôle de nombreuses fonctions endocriniennes et neurovégétatives ; (iii) une activation du gyrus frontal inférieur droit et de la tête du noyau caudé droit ; cette dernière structure a été considérée comme une interface limbico-motrice ; l'activation du gyrus frontal inférieur droit et de la tête du noyau caudé droit pourrait être en relation avec les aspects motivationnels de la perception du désir sexuel (cad avec le désir de s'engager soi-même dans une activité sexuelle). En d'autres termes, tant sur le plan des phénomènes psychologiques perçus par les sujets que sur le plan des activations neurales, l'excitation sexuelle paraît comporter : (i) un aspect perceptivo-cognitif qui permet l'évaluation et l'interprétation du stimulus présenté; un aspect motivationnel-conatif, préparant un éventuel pattern d'action moteur dirigé vers le monde extérieur; et une réponse interne -- émotionnelle, endocrinienne et neurovégétative -- préparant l'organisme à une telle activité dirigée vers le monde extérieur.

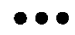

\section{Rôle de la Dopamine dans le contrôle de l'érection et du com- portement sexuel chez le rat}

\author{
E.M. HULL \\ Dept Psychology, SUNY, Buffalo, NY \\ 14260-4110, USA.
}

L'aire préoptique médiane de l'hypothalamus est un site d'intégration majeur intervenant dans le contrôle du comportement sexuel chez tous les Vertébrés mâles. Les agonistes dopaminergiques ont été proposés pour traiter les dysfonctions érectiles chez l'homme, et ils facilitent l'érection chez le rat. Dans cette espèce, la rétraction tonique du prépuce provoque l'apparition d'érections réflexes sous la forme d'épisodes d'érections plus ou moins intenses du gland et des corps caverneux. Les érections les plus intenses des corps caverneux sont appelées dorsiflexions. L'aire préoptique médiane, cible de projections dopaminergiques issues du système dopaminergique périventriculaire, pourrait être la cible des agonistes dopaminergiques facilitateurs des réflexes sexuels. Il semble exister trois effets distincts de la dopamine sur les réflexes sexuels. D'abord, un mécanisme à bas seuil désinhiberait les érections réflexes et les dorsiflexions. Des microinjections de très faibles doses d'un agoniste des récepteurs dopaminergiques D2 et D3 (appartenant tous deux à la famille des récepteurs D2) dans l'aire préoptique médiane raccourcissent la latence de la première érection ou dorsiflexion, mais ne modifient pas le nombre de réponses réflexes ou d'émissions séminales. En revanche des microinjections d'un agoniste des récepteurs dopaminergiques D1 n'ont pas d'effet sur la latence des réflexes. Par conséquent le contrôle à bas seuil des réflexes sexuels est médié par des récepteurs dopaminergiques de la famille D2. Des microinjections de faibles doses d'apomorphine, agoniste dopaminergique D1/D2, raccourcissent également la latence de la première érection réflexe. Cependant l'injection simultanée d'un antagoniste des récepteurs dopaminergiques D1 ou D2 et d'apomorphine bloque partiellement l'effet de l'apomorphine. Ceci suggère que, en plus de la levée d'inhibition produite principalement par la stimulation de récepteurs dopaminergiques D2, il doit exister une 
activité de fond, médiée par des récepteurs dopaminergiques D1 pour permettre l'expression de l'effet D2. Les faibles doses d'agonistes dopaminergiques D2 modifient seulement la latence et pas le nombre des érections réflexes. Le mécanisme à bas seuil médié par les récepteurs dopaminergiques D2 semble donc correspondre à une levée d'inhibition.

Le second mécanisme, de seuil intermédiaire, augmente le nombre d'érections réflexes et de dorsiflexions, et serait dû à la mise en jeu des récepteurs dopaminergiques D1. Des microinjections soit de tétrahydrothienopyridine, agoniste des récepteurs dopaminergiques $\mathrm{D} 1$, soit de doses modérées d'apomorphine dans l'aire préoptique médiane augmentent le nombre d'érections réflexes et de dorsiflexions. Les effets facilitateurs dûs à l'apomorphine sont abolis si l'on a réalisé auparavant des microinjections d'un antagoniste des récepteurs dopaminergiques $\mathrm{D} 1$. La stimulation des récepteurs D1 inhibe également l'éjaculation. Il semble que le rôle de la dopamine dans l'aire préoptique médiane soit d'augmenter la réaction à des stimuli, plutôt que d'activer directement les voies nerveuses proérectiles. L'érection apparaîtrait ainsi plus facilement en présence d'une femelle réceptive au cours d'un test de copulation, ou en réponse réflexe à la rétraction du prépuce.

Le troisième mécanisme est à haut seuil et orienterait l'équilibre du contrôle des fonctions sexuelles par le système nerveux autonome en faveur de l'émission séminale, laquelle est médiée par le système nerveux sympathique. Des microinjections de fortes doses de quinerolane, agoniste des récepteurs dopaminergiques D2/D3, ou d'apomorphine dans l'aire préoptique médiane augmentent le nombre d'émissions séminales mesurées ex copula. On bloque cette augmentation par des injections préalables de raclopride, antagoniste des récepteurs D2. De plus, le quinerolane inhibe les érections et les dorsiflexions. Ceci peut expli- quer la période réfractaire suivant une éjaculation.

Pour vérifier si ces produits modifient le comportement sexuel, des microinjections d'agonistes et d'antagonistes des récepteurs D1 et D2 ont été réalisées dans l'aire préoptique médiane juste avant des tests de copulation. L'agoniste D1 augmente la fréquence de copulation, augmentant par là-même le nombre d'éjaculations au cours d'un test de 30 minutes. Cet effet est bloqué par un antagoniste des récepteurs D1. Des microinjections de fortes doses d'un agoniste des récepteurs D2/D3 retardent la copulation (comme si les rats étaient en période réfractaire post-éjaculatoire) puis ensuite réduisent le nombre d'intromissions précédant une éjaculation (provoquant une éjaculation "prématurée").

En résumé, la dopamine libérée dans l'aire préoptique médiane immédiatement avant et pendant la copulation lève l'inhibition exercée sur les réflexes sexuels en agissant sur des récepteurs dopaminergiques D2 à bas seuil. De plus forts niveaux de dopamine, agissant sur des récepteurs de type D1, potentialisent les effets de stimuli à l'origine des érections et des dorsiflexions. De plus l'éjaculation est retardée. Des niveaux élevés de dopamine permettent à celle-ci d'agir sur des récepteurs de la famille D2 à haut seuil, faisant basculer la régulation autonome des fonctions sexuelles en faveur de l'éjaculation et inhibant l'érection. Il est probable que les récepteurs D2 à bas seuil et les récepteurs D2 à haut seuil représentent deux sous-types différents de la même famille. On peut penser que la levée précoce de l'inhibition exercée sur les réflexes sexuels est médiée par des récepteurs à bas seuil de type D3, parce que la dopamine a plus d'affinité pour ces derniers que pour les récepteurs D2. Par conséquent la dopamine, agissant dans l'aire préoptique médiane, participerait à l'évolution temporelle des différentes étapes de la copulation, ainsi qu'à son efficacité. 


\section{Rôle du noyau paraventriculaire de l'hypothalamus dans la com- mande de l'érection}

\author{
A. ARgiolas \\ Bernard B Brodie Dept Neuroscience, Univ \\ Cagliari, Cagliari, Italie.
}

L'érection apparaît dans différentes situations, en particulier au cours de la copulation, pendant le sommeil paradoxal ou en réponse à des fantasmes. On peut envisager que différents mécanismes nerveux et endocriniens régulant l'érection sont mis en jeu, en fonction de la situation au cours de laquelle l'érection apparaît. Au sein du système nerveux central, plusieurs structures nerveuses centrales participent à la commande de l'érection. Parmi celles-ci, le noyau paraventriculaire de l'hypothalamus (PVN) a fait l'objet d'études détaillées. On pense aujourd'hui, à partir d'études morphologiques et fonctionnelles principalement menées chez le rat, qu'une population de neurones ocytocinergiques (réalisant la synthèse et la libération du neuropeptide ocytocine) dont les corps cellulaires sont présents dans le PVN et dont les prolongements axonaux gagnent l'hippocampe, le bulbe rachidien et la moelle épinière, joue un rôle important dans la commande de l'érection. Ces neurones ocytocinergiques provoqueraient l'érection lorsqu'ils sont excités par différents neuromédiateurs tels que l'ocytocine elle-même, par l'intermédiaire de récepteurs ocytocinergiques voisins de ceux identifiés dans l'utérus, par la dopamine et les agonistes dopaminergiques par la mise en jeu de récepteurs D2, et par des acides aminés excitateurs agissant sur des récepteurs de type NMDA (N-méthyl-Daspartate). A l'opposé, la mise en jeu de ces neurones par l'ocytocine, la dopamine et les récepteurs de type NMDA est abolie en présence de morphine agissant sur des récepteurs aux opiacées de type $\mu$. La morphine abolit également les érections provoquées par des microinjections de ces agents dans le PVN.

L'effet de la dopamine, de l'ocytocine et des acides aminés excitateurs se traduirait par une augmentation de calcium intracellulaire dans les neurones ocytocinergiques du PVN. L'omega-conotoxine, puissant bloqueur des canaux calciques de type $\mathrm{N}$, abolit toute érection induite par la microinjection d'agonistes dopaminergiques D2 ou d'ocytocine dans le PVN. L'érection induite par la microinjection de NMDA est abolie par le MK-801, un antagoniste non compétitif puissant des canaux calciques couplés aux récepteurs de type NMDA.

L'augmentation de calcium intracellulaire activerait la monoxyde d'azote synthase (NOS), enzyme calcium-calmoduline dépendante, dans le corps cellulaire des neurones ocytocinergiques contrôlant l'érection. En effet, l'érection induite par les agents cités ci-dessus est abolie après injection intraPVN d'inhibiteurs de la NOS, et à l'aide de la microdialyse intracérébrale on a montré que les agonistes dopaminergiques, l'ocytocine et le NMDA délivrés aux doses qui provoquent des érections augmentaient la production de monoxyde d'azote (NO) dans le PVN. De plus l'injection de donneurs de NO dans le PVN provoque des érections tout à fait comparables à celles induites par les agonistes dopaminergiques, l'ocytocine et le NMDA. Enfinf la NOS a été mise en évidence dans les neurones ocytocinergiques du PVN.

La mise en jeu de la NOS dans le contrôle de l'érection par le PVN est également suggérée par d'autres expériences. Chez des rats mâles qui ne copulent pas en présence de femelles réceptives, mâles qualifiés d'impuissants, on a montré que la quantité des ARN messagers codant pour la NOS était réduite de moitié par rapport à celle mesurée chez des rats copulant, cette différence n'étant présente que dans le PVN. Par des techniques de microdialyse, on a également montré que la production de NO augmente 
dans le PVN de rats mâles en présence de femelles réceptives, plus qu'en présence de femelles non réceptives, même si l'on empêche tout contact sexuel. La production de NO dans le PVN augmente davantage si les mâles copulent. Un parallèle est donc établi entre le niveau d'excitation sexuelle et la production de NO dans le PVN. Il ne faut cependant pas oublier que le PVN n'est probablement pas la seule structure nerveuse centrale participant au contrôle de l'érection. En particulier on a montré un rôle proérectile des peptides de la famille de l'ACTH/MSH, comme des agonistes des sous-types 5-HT2C des récepteurs sérotoninergiques. Or ces derniers agents sont inefficaces lorsqu'ils sont délivrés dans le PVN.

\section{Régulation médullaire : Rôle de l'ocytocine}

\author{
O. RAMPIN
}

Laboratoire de Neurobiologie des Fonctions Végétatives, INRA, F-78352 Jouy-en-Josas Cedex.

\section{La moelle épinière contient les moto- neurones autonomes et somatiques contrôlant les mécanismes locaux de l'érection}

Les mécanismes locaux de l'érection pénienne reposent sur la relaxation des fibres musculaires lisses du tissu érectile des corps spongieux et caverneux et des artères à destinée pénienne. Cette relaxation est commandée par le système nerveux autonome. L'innervation parasympathique proérectile prend son origine dans la colonne intermédiolatérale de la moelle sacrée (étages S2-S4 chez l'homme) et gagne le tissu érectile par les nerfs pelviens et caverneux. L'innervation sympathique antiérectile, issue des neurones de la colonne intermédiolatérale de la moelle thoracolombaire (étages Tll-L2 chez l'homme), gagne le pénis par la chaîne sympathique paravertébrale et les nerfs honteux. Un second contingent sympathique a destinée pénienne, issu de la commissure grise dorsale des mêmes étages thoracolombaires, chemine dans le nerf hypogastrique puis le nerf caverneux. Les muscles striés bulbospongieux et ischiocaverneux, circonscrivant respectivement la racine des corps spongieux et caverneux, participent également à la survenue de l'érection. Leur contraction, commandée par les motoneurones du nerf honteux, augmente considérablement la rigidité pénienne.

Les structures supraspinales modulent l'activité des centres spinaux de l'érection dans différentes situations

L'érection survient en réponse a des stimulations génitales, a des fantasmes, au cours du sommeil paradoxal, durant le coït ... Ainsi différentes structures au sein du système nerveux central sont activées par des informations afférentes d'origine centrale et périphérique et commandent la survenue d'érections dans une situation déterminée. Il est logique de postuler que l'érection résulte à la fois de l'activation du centre parasympathique sacré et de l'inhibition du centre sympathique thoracolombaire. Le rôle des stuctures supraspinales dans la nécessaire coordination de ces centres médullaires autonomes demeure mal connu. Plusieurs études récentes ont montré que la stimulation de noyaux hypothalamiques tels que l'aire préoptique médiane ou le noyau paraventriculaire provoquait des érections chez des rats éveillés ou anesthésiés. On a également montré que les projections raphé-spinales sérotoninergiques exerçaient une inhibition tonique sur les érections réflexes. Chez le rat, ces dernières sont plus faciles à provoquer après section médullaire thoracique, aprés lésion chimique ou électrolytique des noyaux du raphé, en particulier du noyau paragigantocellulaire, celui-ci projetant 
directement vers les centres médullaires sacrés. Enfin les érections apparaissant au cours du sommeil paradoxal chez le rat sont abolies après des sections réalisées à l'étage mésencéphalique

Les neurones parvocellulaires du noyau paraventriculaire de l'hypothalamus sont à l'origine de projections descendantes vers la moelle épinière

Le groupe des neurones parvocellulaires du noyau paraventriculaire de l'hypothalamus émet des prolongements vers le bulbe rachidien et la moelle épinière. La présence de fibres ocytocinergiques a été démontrée à tous les étages de la moelle épinière. Ces fibres sont présentes principalement dans la corne dorsale, mais également dans la commissure grise dorsale, le noyau intercalé et la colonne intermédiolatérale. La corne ventrale reçoit peu de fibres ocytocinergiques. La distribution des sites de liaison au récepteur de l'ocytocine se superpose aux aires dans lesquelles les fibres ocytocinergiques sont présentes. Des contacts synaptiques entre fibres ocytocinergiques et neurones préganglionnaires sympathiques ont été mis en évidence.

Plusieurs arguments plaident en faveur du rôle des projections hypothalamospinales ocytocinergiques dans la régulation de l'érection

La stimulation du noyau paraventriculaire augmente la libération d'ocytocine dans la moelle épinière chez le rat anesthésié. L'ocytocine gagne la moelle épinière par transport axonal. Les taux d'ocytocine mesurés dans le liquide céphalorachidien augmentent d'un facteur 2 à 3 au cours des 30 minutes qui suivent l'éjaculation chez le rat. La lésion de la partie parvocellulaire du noyau paraventriculaire abolit cette augmentation. On a également montré que la stimulation pharmacologique du noyau paraventriculaire par injection d'ocytocine, de dopamine, de glutamate ou de donneurs de monoxyde d'azote provoquait des épi- sodes d'érection chez le rat éveillé. Enfin la lésion du noyau paraventriculaire réduit le nombre de rats présentant des érections réflexes et psychogéniques.

Des données expérimentales récentes montrent que l'ocytocine, libérée dans les étages lombosacrés de la moelle épinière, est un neuropeptide régulateur de l'érection chez le rat

Les travaux récents issus d'une collaboration entre plusieurs laboratoires* démontrent la présence de contacts synaptiques entre fibres ocytocinergiques et neurones préganglionaires parasympathiques sacrés identifiés par transport axonal rétrograde à partir du nerf pelvien. Des récepteurs à l'ocytocine ont été mis en évidence dans les couches superficielles de la corne dorsale de la moelle aux étages lombosacrés, et également dans le plan intermédiaire, principalement la commissure grise dorsale et la colonne intermédiolatérale. Des injections intrathécales d'ocytocine ont été réalisées chez le rat anesthésié. Seules les injections d'ocytocine en regard des étages lombosacrés (L5-L6) de la moelle ont provoqué des réponses érectiles, attestées par des augmentations de pression intracaverneuse. Le nombre, l'amplitude et la surface de ces réponses érectiles étaient dose-dépendants. Des injections du véhicule $(\mathrm{NaC1} 0,9 \%)$ ou d'arginine-vasopressine en regard des mêmes étages médullaires, comme l'injection d'ocytocine en regard des étages TllT12 n'avaient pas d'effet. Des réponses érectiles étaient également enregistrées lorsque la [Thr4, Gly7] ocytocine, agoniste spécifique de l'ocytocine, était injectée. L'injection préalable d'un inhibiteur compétitif de l'ocytocine supprimait tout effet proérectile de l'ocytocine.

\section{Conclusions et perspectives}

Ces approches expérimentales démontrent le rôle proérectile des projections hypothalamospinales ocytocinergiques. Au plan expérimental, la contribution de ces projec- 
tions à la survenue des érections dans différentes situations reste à explorer. On peut facilement envisager l'importance de leur rôle dans les érections survenant au cours de la copulation et leur faible effet dans la modulation des érections réflexes. Les résultats récents apportent une démonstration morphologique et fonctionnelle pour expliquer les effets proérectiles des stimulations du noyau paraventriculaire chez le rat éveillé. La recherche d'agonistes spécifiques des récepteurs ocytocinergiques doit être envisagée dans une approche thérapeutique des dysfonctions érectiles liées à la commande spinale de l'érection. Il faudra cependant expertiser le rôle de l'ocytocine dans la régulation spinale des autres fonctions pelviennes.

\section{* Laboratoire de Neurobiologie des Fonctions Végétatives, I.N.RA., Jouy-en-Josas, Groupe de Recherche en Urologie, Faculté de Médecine Paris-Sud, Le Kremlin-Bicêtre, IDN-CNRS URA 1488, Université Paris 6 et Laboratoire de Phy- siologie Générale, CNRS UR4 1446, Université Louis Pasteur, Strasbourg. Ces travaux ont béné- ficié d'un soutien financier de l'Institut pour la Recherche sur la Moelle Epinière.}

\section{Conséquences sexuelles des traumatismes médulaires}

\section{Les greffes médullaires de neurones : Données expérimentales}

\author{
A. PRIVAT \\ U.336 INSERM Montpellier
}

Les lésions médullaires graves entraînent des déficits de la sensibilité et de la commande de la musculature squelettique, qui sont souvent au premier plan du tableau clinique. Toutefois, les conséquences au niveau de la sphére uro-génitale s'avèrerent souvent les plus invalidantes, en particulier pour les paraplégiques bas. Nous avons étu- dié chez le rat mâle adulte la conséquence sur la séquence érection-éjaculation d'une section médullaire complète réalisée au niveau D7-D8. Chez le rat témoin non anesthésié, entravé, la stimulation penienne se traduit par une séquence érection-éjaculation dont la fréquence peut être quantifiée. La commande sérotonergique est affirmée par l'influence positive d'un traitement préalable par un inhibiteur de la réabsorption de la sérotonine, la zimélidine (Mas et coll., 1985 ; Blier et De Montigny, 1983). Chez le rat paraplégique, l'érection est plus fréquente, mais n'est pas suivie d'éjaculation. La transplantation, sous le niveau de la lésion, de neurones embryonnaires sérotonergiques rétablit sur le plan anatomique l'innervation sérotonergique détruite par la dégénérescence wallérienne qui suit la section.

Un mois après la transplantation, l'injection de zimélidine suivie d'une stimulation pénienne rétablit la séquence érection-éjaculation, alors que les rats lésés non transplantés ne montrent pas d'éjaculation. Nous pouvons ainsi affirmer la commande sérotonergique médullaire de l'éjaculation chez le rat. Ce réflexe absent chez le rat paraplégique est rétabli après transplantation sérotonique, comme est rétablie la locomotion réflexe (Yakovleff et coll, 1996 ; Feraboli-Lenherr et coll, 1997).

\section{Le tractus séminal des patients blessés médullaires}

\author{
J.C. SOUFIR
}

Laboratoire de Biologie andrologique. $\mathrm{CHU}$ Bicêtre.

L'examen de sperme permet une exploration directe de l'appareil génital. Il est possible d'évaluer (1) la fonction testiculaire (spermatogénèse) en déterminant le 
nombre et la morphologie des spermatozoïdes et (2) la fonction des différents organes du tractus génital - épididymes, prostate et vésicules séminales - en dosant des marqueurs spécifiques séminaux.

Pour obtenir chez les paraplégiques des éjaculats contenant des spermatozoïdes, on est appelé à utiliser, en fonction du niveau lésionnel, deux types de méthodes : vibromassage lorsque l'arc réflexe D12-L1 est fonctionnel, électroéjaculation lorsqu'il ne l'est pas.

La comparaison des spermogrammes obtenus par ces deux techniques (vibromassage, nombre de patients, $\mathrm{n}=17$; électrostimulation, $n=12$ ) met en évidence que, dans les deux groupes de patients, la concentration et la morphologie des spermatozoïdes sont relativement peu affectées (vibromassage : $101 \pm 7$; électrostimulation : $103 \pm 23$ millions par $\mathrm{ml}$; formes typiques respectivement 34 et $38 \%$ ) ; par contre, on observe dans les deux groupes, une atteinte fonctionnelle - \% élevé de spermatozoïdes immobiles - qui est beaucoup plus importante chez les patients dont l'ejjaculat a été obtenu par électrostimulation (vibromassage $=35 \%$; électrostimulation $=9 \%$ ).

La mesure dans le sperme d'index biochimiques témoignant de la maturation épididymaire des spermatozoïdes (L-carnitine libre) ou de leur protection contre la lipopéroxydation (fructose pour les vésicules séminales, citrate pour la sécrétion prostatique) fournit des é1éments permettant d'expliquer le déficit de mobilité : dans les spermes obtenus par vibromassage, on note une diminution marquée de la sécrétion épididymaire $(-65 \%)$ et une réduction plus faible de la sécrétion prostato-vésiculaire (citrate :-15\%, fructose : $-40 \%$ ) ; dans les spermes obtenus par électrostimulation, la sécrétion épididymaire est inexistante et la sécrétion prostato-vésiculaire très réduite (citrate : $-70 \%$, fructose : $-80 \%$ ).

Il apparaît ainsi que, chez les paraplégiques, l'atteinte génitale concerne plus les organes responsables de la maturation, de la conservation et de la protection des gamètes (épididymes ainsi que probalement prostate et vésicules séminales) que les testicules.

Participants à cette étude

Cytologie : A. Soumah, P. Jouannet - Biochimie du sperme : P. Weber, J.C. Soufir

Neurologues : N. François, C. Fakacs, M. Maury

\section{Le recours à I'A.M.P. chez les patients bilessés médullaires}

\section{(L'expérience de Bicêtre)}

\author{
V. IZARD
}

Service d'Urologie, CHU de Bicêtre

Le blessé médullaire reste moins lontemps dans les centres. Il s'autonomise plus rapidement pour mieux se réinsérer dans la société et une collaboration multidisciplinaire de différents spécialistes permet de mieux répondre à ses aspirations. L'andrologue est désormais pressenti tôt dans l'histoire du handicap ; on exige de lui un savoir-faire adapté au cheminement de l'individu, qu'il s'agisse de troubles sexuels, d'un projet d'enfant, ou des deux à la fois.

Un nombre important d'hommes blessés médullaires parviennent à procréer à partir de sperme éjaculé recueilli naturellement, ou à l'aide d'une stimulation par vibromassage ou d'une stimulation électrique (réalisée dans notre équipe par Noël François).

Lorsqu'un éjaculat ne peut être obtenu ou que les spermatozoïdes $(\mathrm{Spz})$ éjaculés ne peuvent être utilisés à la lueur des progrès de l'assistance médicale à la procréation (AMP), le recours au prélèvement chirurgical de gamètes est indiqué. 
Entre 1986 et 1997, 39 patients blessés médullaires nous ont été confiés, totalisant 53 tentatives d'AMP, chez des sujets adressés après tentative infructueuse de recueil de spermatozoïdes éjaculés.

Les prises en charges des couples ont été effectuées essentiellement avec les Laboratoires de Biologie de la reproduction et les Services de Gynécologie-Obstétrique de 1'Hôpital Antoine Béclère, de 1'Institut Mutualiste Montsouris (HIUP) ou des Maternités Cochin Baudelocque III et Saint-Vincent-de-Pau1 .

L'historique des modalités d'interaction gamétique (53 tentatives) a suivi l'évolution du savoir-faire des biologistes et cliniciens gynécologues :

- insémination intra-utérine (IIU) 10 fois

- insémination in-vitro classique (FIV) 15 fois

- injection sous-zonale de quelques Spz (SuZI) 19 fois

- injection intra-cytoplasmique d'un Spz (ICSI) 9 fois

Quelques grossesses intra-utérines ont été observées, selon les modes d'interaction :

IIU : 0

FIV : 31 fausse couche et 2 naissances

SuZI: 11 naissance

ICSI: 41 fausse couche et 3 naissances attendues

Aucune grossesse n'a été obtenue après IIU chez 8 couples totalisant dix tentatives synchrones du recueil de spermatozoïdes.

Le développement des techniques de fécondation assistée a fait préférer la ponction folliculaire ovarienne avec replacement d'embryons dès $1990 ; 8$ grossesses ont été ainsi obtenues en AMP avec naissance de 3 enfants pour 3 couples, grossesse en cours à ce jour avec attente d'un enfant pour 2 couples et attente de jumeaux pour un couple ...
L'effectif des 39 blessés médullaires constitue le sous-groupe le plus important des 52 patients présentant une infécondité par anéjaculation organique traités à Bicêtre pendant cette période. Par comparaison, les 13 patients non blessés médullaires avec anéjaculation post-opératoire [5 cas], neuropathie diabétique (4 cas), spina bifida ( 1 cas), médullopathie parasitaire (1 cas d'hydatidose), médullopathie vasculaire (1 cas) ou atonie de la voie séminale profonde (1 cas) ont eu des résultats meilleurs en terme de conception avec 9 grossesses réparties dans ces 13 couples et naissance d'un enfant pour 7 d'entre-eux.

Dans notre série, les résultats de la fécondation in-vitro sont médiocres chez les blessés médullaires. Les résultats actuels de I'ICSI paraissent encourageants, ce qui confirme les données de la littérature qui prône des recueils de plus en plus proximaux, volontiers testiculaires dans ces situations.

D'autres facteurs peuvent intervenir dans les résultats récents de notre équipe, tels que l'attention toute particulière dans la préparation au geste chirurgical, cas par cas, le rapprochement des auto-sondages, l'éducation visant à éviter l'hypertermie scrotale préopératoire, la désinfection soigneuse du scrotum, et le soin particulier apporté par les gynécologues à la préparation de la femme ...

Le mode d'éventuelle analgésie, lié à la médullopathie, a peu changé sur la décennie; la technique et le lieu de prélèvement chirurgical de gamètes ont évolué mais s'adaptent à chaque situation, à chaque côté droit/gauche :

- ponction du déférent juxta-épididymaire

- aspiration/succion du canal épididymaire

- aspiration/succion de l'apex d'un cône efférent, juxta-testiculaire

- prélèvement biopsique de pulpe testiculaire. 
Dès 1991 , les biologistes de notre équipe ont su saisir l'opportunité d'une congélation éventuelle des gamètes non utilisées, quelle que soit la technique de recueil chirurgical employée. Depuis lors, l'expérience des équipes ayant une grande pratique de l'ICSI a montré que les spermatozoïdes épididymaires ou testiculaires décongelés donnaient des résultats identiques à ceux des spermatozoïdes utilisés frais, en nombre de fécondations obtenues.

L'expérience de Bicêtre bénéficie, nous l'espérons, d'une approche conviviale globaliste de la sexualité du blessé médullaire. La prise en charge du projet de procréation profite des dernières techniques de I'AMP et s'adapte aux connaissances du monde du handicap.

Face au projet de procréation du couple, l'approche multidisciplinaire tient aussi compte de la situation de la compagne, souvent sous-évaluée avant la tentative biologique, et qui devra vivre au mieux l'échec et le succès ... Au stade précoce de la blessure médullaire, la collaboration avec les praticiens spécialisés dans le handicap se profile pour mieux définir un moment licite optimal de recueil invasif de gamètes pour cryopréservation, dans un souci délicat d'efficacité biologique future et d'innocuité présente, chez un blessé consultant à l'heure du deuil du handicap ...

10

\section{Actualité du traitement pharmacologique des troubles de l'érection chez les blessés médullaires}

F. Giuliano

Service d'Urologie CHU de Bicêtre

La découverte de l'effet érectogène de l'admnistration intracaverneuse de sub- stances pharmacologiques en 1982-83 a bouleversé la prise en charge thérapeutique des dysfonctions érectiles quelle qu'en soit l'étiologie Il s'agissait initialement de la papavérine, inhibiteur non spécifique des phosphodiestérases, et de la phentolamine, antagoniste des récepteurs alpha-adrénergiques.

Parallèlement de nombreuses recherches pharmacologiques visant à décrire les mécanismes extra et intracellulaires de la relaxation des fibres musculaires lisses du tissu érectile des corps spongieux et caverneux, événement clé de l'érection, ont été conduites depuis quinze ans [1]. S'appuyant sur des travaux expérimentaux in vitro et in vivo chez l'animal, un grand nombre de substances pharmacologiques, délivrées par voie intracaverneuse, ont fait l'objet d'études cliniques dans cette indication. Il faut retenir l'efficacité régulière de quelques-unes de ces substances, qu'il est nécessaire de subdiviser en deux groupes [2] : 1) les drogues facilitatrices, capables de provoquer le plus souvent une tumescence plutôt qu'une érection rigide lors d'une injection intracaverneuse médicalisée, c'est à dire réalisée par le praticien 2) les drogues érectogènes, dont l'administration intracaverneuse dans les mêmes conditions provoque le plus souvent une érection suffisamment rigide pour permettre la pénétration.

Le premier groupe comprend a) le vasoactive intestinal peptide (VIP), neuromédiateur non adrénergique non cholinergique, libéré par les terminaisons nerveuses parasympathiques caverneuses proérectiles, b) le calcitonin gene related peptide (CGRP) également présent naturellement dans le tissu érectile, neuromédiateur libéré par les terminaisons sensitives agissant directement sur les fibres musculaires lisses ou interagissant avec les terminaisons nerveuses autonomes efférentes, c) le linsidomine ou Sinl, donneur par un mécanisme non enzymatique de monoxyde d'azote (NO) considé- 
ré comme le principal agent responsable de la relaxation musculaire lisse érectile, dont l'origine est double chez l'homme: nerveuse et endothéliale, faisant intervenir dans ce dernier cas, lors de l'afflux de sang artériel inaugurant l'érection, une stimulation mécanique ou shear stress des cellules endothéliales bordant les espaces sinusoïdes, d) la phentolamine déjà mentionnée, e) le moxisylite, antagoniste possédant une certaine sélectivité pour les récepteurs adrénergiques de sous-type alpha 1 , il s'agit de la seule substance de ce groupe ayant à ce jour obtenu l'A.M.M. en France dans cette indication.

Le groupe des substances érectogènes comprend le chlorhydrate de papavérine, alcaloïde de l'opium, citée plus haut et la prostaglandine $\mathrm{El}$, possédant également l'A.M.M. en France, dont l'action repose sur une diminution de la libération de noradrénaline par un mécanisme présynaptique et très probablement sur une activation de récepteurs membranaires spécifiques médiée par une activation de l'adénylate cyclase intracellulaire.

Les études cliniques ont bien décrit l'intérêt des injections intracaverneuses chez les patients blessés médullaires [3-6]. Il s'agit d'un traitement symptomatique efficace chez la grande majorité des patients. Les effets secondaires à type de priapisme sont d'autant plus rares que l'éducation des patients a été attentive, la survenue d'une fibrose intracaverneuse localisée est peu fréquente. L'aide de la partenaire pour la réalisation des injections est requise chez les patients tétraplégiques. Ainsi cette approche thérapeutique a-t-elle dans de nombreux cas supplanté l'implantation chirurgicale de prothèses péniennes volontiers pratiquée dans un passé proche.

L'absorption percutanée de substances pharmacoloqiques appliquées sur le revêtement cutané pénien, scrotal et périnéal a été démontrée. Quelques essais cliniques préliminaires ont conclu à une certaine effi- cacité de la délivrance par cette voie de papavérine et de prostaglandine $\mathrm{El}$ chez les patients blessés médullaires $[7,8]$. La poursuite de la recherche pharmacologique (associations de molécules proérectiles, combinaisons avec des substances favorisant le passage cutané ) devrait permettre d'améliorer les résultats de cette approche locale "non invasive".

La voie d'administration intra-urétrale a également été développée. Il s'agit de déposer dans la portion distale de l'urètre, à l'aide d'un applicateur (systéme MUSE), une substance pharmacologique dont le conditionnement permet sa diffusion vers le tissu érectile des corps spongieux et caverneux. Des résultats très prometteurs ont été rapportés [9] lors de la délivrance par cette voie de prostaglandine El chez des patients souffrant de troubles de l'érection considérés d'origine organique à l'exception des patients blessés médullaires. Chez ces derniers, l'efficacité d'une telle approche thérapeutique est très probable, une étude spécifique est cependant requise considérant les caractéristiques spécifiques de ces patients en particulier urétrales (autosondages).

L'avancée thérapeutique majeure est désormais représentée par des résultats préliminaires concernant l'efficacité de la délivrance orale du sildénafil, UK-92,480, inhibiteur des phosphodiestérases de sous-type $\mathrm{V}$ [10]. Ce composé, semblant dépourvu d'effets secondaires importants, inhibe la dégradation du GMPc, second messager intracellulaire du NO, principal substance proérectile, amplifiant ainsi la relaxation musculaire lisse. Une stimulation sexuelle est requise pour l'action de cette substance pharmacologique, la prise devant s'effectuer en règle une heure environ avant le rapport. L'intégrité partielle de la commande nerveuse parasympathique nitrergique pro-érectile est un pré-requis à l'action de ce composé. C'est le cas chez la majorité des patients blessés médullaires qui conservent 
souvent une tumescence voire une rigidité instable en réponse à une stimulation centrale et/ou périphérique $[6,11,12]$.

L'expérience acquise à Bicêtre depuis un an est particulièrement encourageante dans la mesure où des patients paraplégiques antérieurement traités par des auto-injections intracaverneuses sont répondeurs au traitement oral.

D'autres études sont en cours [13], visant à en particulier à affirmer l'effet pro-érectile de composés agissant à l'étage supra-spinal [14], délivrés par voie sublinguale (apomorphine) ou sous-cutanée (analogue de l'alpha-melanocyte-stimulating-hormone) mais n'ont pas concerné jusqu' à ce jour de patients blessés médullaires.

Ainsi la prise en charge pharmacologique des dysfonctions érectiles consécutives aux lésions de la moelle épinière a formidablement progressé. La cible pharmacologique médullaire demeure inexplorée [15]. Nul doute qu'elle représente pour l'avenir un nouveau champ d'investigation de thérapeutiques innovantes à distance de la lésion mais également plus précocément. L'effort doit désormais porter également sur l'autre grande fonction de reproduction, l'éjaculation, fréquemment altérée dans ce contexte traumatique.

\section{Références}

1. Giuliano F., Rampin O., Benoit G., Jardin A. : Pharmacologie périphérique de l'érection. Progrès en Urologie 1997, $7: 24-33$.

2. Giuliano F., Blanchet P., Benoit G., Jardin A. : Place des injections intracaverneuses dans le diagnostic et le traitement des troubles de l'érection. Presse Med. 1994, 23 : 1257-1262.

3. Courtois F., Charvier K., Belley C., Lenche A., Monet F., LeduC B., RAYMond D. : Les injections intracaverneuses et l'approche sexologique auprès des hommes paraplégiques et tétraplégiques. Revue Sexologique 1995, 3, 2.

4. Costa P., Bresssolle F., Sarrazin B., Mosser J., Navratil H., Galtier M. : Moxisylite plasma kinetics in humans after intracavernous administration. Biopharmaceutics \& Drug Disposition 1992,715, 1-9.
5. Padma-Nathan H., Kanelios A. : The management of erectile dysfunction following spinal cord injury. Seminars in Urology 1992, 10 (2), 133-137.

6. SMITH E.M., BoDNER D.R. : Sexual dysfunction after spinal cord injury. Urol. Clin. North Am. 1993, 3, 535-542.

7. KIM E.D., EL-Rashidy R., MC VARY K.T. : Papaverine topical gel for treatment of erectile dysfunction. J.Urol. 1995,153, 361-365.

8. KrM E.D., MC VARY K.T. : Topical prostaglandin-E 1 for the treatment of erectile dysfunction. J.Urol. 1995,153, 1828-1830.

9. Padma-Nathan H, Hellstrom W.J.G, Kaiser F.E., Labasky R.F., Lue T.F., Nolten W.E., Nonvood P.C., Peterson C.A., Shabsigh R., Tam P.Y., Place V.A., Gesunheit N. : Treatment of men with erectile dysfunction with transurethral alprostadil. N. Engl. J. Med. 1997, 336, 1-7.

10. Glass C., Derry F., Dmsmore W.W., Fraser M., Gardner B.P., MaYtom M.C., ORR M., OSTERloH I.H., SMITH M. : Sildenafil (VIAGRA TM) : an oral agent for men with erectile dysfunction caused by traumatic spinal cord injury- a double-blind, placebo-controlled, single-dose, two-way crossover study using rigiscan. American Spinal Injury Association Mars 24-26, 1997, Houston, TX, USA.

11. Courtois F., Chamer K.F., Leriche A., Raymond D.P., EysetTe M. : Clinical approach to erectile dysfunction in spinal cord injuried men. A review of clinical and experimental data. Paraplegia 1995, $33,628-635$.

12. Giuliano F., Rampin O., Blanchet P., Benoit G., JARDIN A. : Physiologie des troubles de l'érection chez l'homme paraplégique. Andrologie 1994, 2 : 129-132.

13. Giuliano F. : Le traitement per os des troubles de l'érection. Progrès en Urologie 1997, 7 : 108-109.

14. Giuliano F., Rampin O, Benoit G., Jardin A. : Neural control of penile erection. Urol. Clin. North. Am. 1995, 22 : 747-765.

15. Rampin O., Bernabé J., Giuliano F. : Spinal control of penile erection. World J.Urol. 1997, 15 : 2-13. 


\section{La sexualité revisité en 1997 à la lumière de la neurobiologie}

\section{Comparaison des fonctions sexuelles chez le mâle et chez la femelle}

KE MCKENNA

Dept Physiology and Urology, Northwestern Univ, Chicago, IL, USA.

Les recherches portant sur le contrôle nerveux des fonctions sexuelles chez le mâle et la femelle ont tenté d'insister sur les différences entre les sexes. Il est naturel de penser que la différentiation des organes génitaux et de leur fonction demandent des spécialisations du système nerveux qui les commande. La mise en évidence de ce dimorphisme sexuel au sein du système nerveux représente un domaine de recherche très actif. Il faut cependant insister sur le fait que des différences structurales quantitatives ne s'accompagnent pas forcément de différences fonctionnelles. Des exemples de contrôles nerveux différents chez le mâle et la femelle sont parfois proposés, mais l'homologie des fonctions qu'ils contrôlent n'est pas toujours claire (par exemple la lordose chez la femelle et la monte chez le mâle). Les quelques exemples suivants, tirés de la clinique et de l'expérimentation animale, permettront au contraire d'insister sur les ressemblances entre les sexes.

Au cours de l'orgasme, une propriété partagée par les deux sexes est la contraction rythmique des muscles striés du plancher pelvien. L'enregistrement de l'activité électromyographique réalisé chez l'homme a confirmé cette ressemblance : les contractions présentent une grande régularité et l'intervalle qui les sépare augmente au cours du temps. Chez quelques sujets, les contractions régulières sont suivies de contractions de fréquence irrégulière. Les patients sont incapables de reproduire volontairement la haute fréquence et la régularité de ces contractions musculaires du plancher pelvien.

Toujours au cours de l'orgasme, on enregistre une augmentation de la pression artérielle, une tachypnée et une tachycardie dans les deux sexes. L'augmentation et la chute rapides de la fréquence cardiaque ont d'ailleurs été proposées comme témoins objectifs de l'orgasme chez l'homme et l'animal. L'excitation sexuelle, et plus particulièrement l'orgasme, est associée à une augmentation des quantités d'ocytocine plasmatique. L'ensemble de ces données montre que la ressemblance entre les sexes concerne des contrôles nerveux somatique et autonome et des régulations neuroendocriniennes.

Les bases neurophysiologiques de plusieurs aspects des fonctions sexuelles ont été mises en évidence à partir d'études réalisées chez l'animal. On a alors retrouvé des ressemblances et des différences. Les différences majeures concernent le nombre, la taille et la morphologie des afférences et des efférences d'origine pelvienne. Le dimorphisme sexuel était expliqué à l'origine par un effet direct des hormones stéroïdes sexuelles sur les neurones de la moelle épinière. Il est plus vraisemblable que le dimorphisme sexuel observé dans le système nerveux est un effet secondaire de la régulation hormonale des organes cibles. Par exemple l'organisation médullaire de la population des motoneurones du noyau d'Onuf reflète la taille de la masse musculaire périnéale. De même le nombre des afférences sensitives présentes dans le nerf honteux reflète la taille globale des tissus cibles, principalement le pénis et le clitoris. Il faut souligner que malgré la grande différence quantitative du nombre et de la morphologie des neurones pelviens de la moelle lombosacrée qui existe entre mâle et femel- 
le, l'étude des réflexes pelviens n'a pas révélé de grandes différences physiologiques.

La mise en jeu des afférences sensitives d'origine pelvienne chez des rats spinalisés aigus et anesthésiés provoque une réponse très comparable aux contractions rythmiques régulières observées chez l'homme pendant l'orgasme. Cette réponse, que nous avons appelée le réflexe urétrogénital, comprend chez le mâle érection, éjaculation et contraction rythmique des muscles périnéaux. Le réflexe urétrogénital est indépendant du statut hormonal tant chez le mâle que chez la femelle. Le réflexe semble différent chez le mâle et chez la femelle quand on mesure l'activité des organes pelviens, cependant l'activité nerveuse réflexe enregistrée dans les nerfs pelviens est très comparable entre les sexes. La stimulation de l'aire préoptique médiane de l'hypothalamus provoque une réponse urétrogénitale très comparable à la réponse réflexe provoquée par la stimulation des afférences pelviennes chez les deux sexes lorsque la moelle épinière est intacte. Les résultats indiquent qu'une partie au moins des réponses sexuelles réflexes est remarquablement comparable entre mâle et femelle. Des approches pluridisciplinaires associant comportement, physiologie et anatomie sont nécessaires pour mieux comprendre les bases nerveuses des fonctions sexuelles. En particulier il est probablement nécessaire de séparer le comportement sexuel en plusieurs composantes afin d'en étudier son support nerveux.

\section{Psychobiologie de l'excitation sexuelle}

\author{
BD SACHS
}

Dept Psychology, Univ Connecticut, Storrs, USA
Le mot "excitation sexuelle" est généralement employé dans un sens large, par exemple lorsque l'on décrit des études réalisées chez l'animal. Il est souvent confondu avec les notions de motivation et de comportement sexuels. Le développement d'une psychobiologie de l'excitation sexuelle dépend de l'usage précis de ce terme, d'une définition qui s'appliquerait aussi bien à l'homme qu'à l'animal, et à la mise au point de modèles animaux appropriés. Une revue de la littérature concernant la sexualité de l'homme suggère que l'érection est l'indice le plus fiable et le plus plausible de l'excitation sexuelle, indice également présent chez de nombreuses espèces animales. Cependant les auteurs négligent trop souvent la situation au cours de laquelle l'érection apparaît ou n'apparaît pas. Cet état d'esprit est particulièrement malheureux dans le cas des "faux positifs", par exemple des érections apparaissant au cours du sommeil, et si l'on accepte le fait que la régulation physiologique de l'érection varie selon la situation au cours de laquelle elle apparaît. Nous avons récemment mis en évidence l'apparition d'érections chez des rats mis en présence de femelles réceptives auxquelles ces mâles n'avaient pas accès. Ces érections "sans contact" chez le rat sont analogues des érections psychogéniques chez l'homme. Les érections sans contact représentent le premier modèle pratique développé chez l'animal de laboratoire pour étudier l'excitation sexuelle. Ce modèle ne doit pas être confondu avec l'activité motrice requise par la copulation ni avec les stimulations somatosensorielles qui sont présentes au cours du coït. Les conclusions de nos travaux récents confirment l'intérêt de notre modèle pour l'étude de l'excitation sexuelle $: 1$. Des mâles qui ne copulent plus après une lésion de l'aire préoptique médiane de l'hypothalamus sont cependant excités sexuellement par des femelles en oestrus. 2. Il existe une double dissociation entre les structures cérébrales nécessaires à l'ex- 
pression des érections sans contact et celles nécessaires à la copulation. 3 . Des stimuli aversifs associés à la présentation de femelles en oestrus inaccessibles suppriment les érections sans contact, mais ont peu ou pas d'effet sur la copulation, ou sur les érections pendant la copulation, lorsque les mâles ont accès aux femelles.

0

\section{Le point de vue du neuro- endocrinologiste}

\author{
J.D. VINCENT
}

Institut Alfred Fessard-CNRS, Gif sur Yvette

Les fonctions sexuelles se dissimulent au coeur de l'hypothalamus. Nous y retrouvons le principe de dualité qui fait cohabiter chez un individu, à quelques neurones de distance dans le même coin d'encéphale, un centre mâle et un centre femelle. Ces centres ne fonctionnent pas à la façon d'un interrupteur ouvert ou fermé, leur seuil d'activation varie, notamment selon le taux des stéroïdes sexuels dans le sang. Il existe en outre des relations de réciprocité entre les composants mâles et femelles. Ces faits ont été établis à la suite d'expériences de lésions ou de stimulations électriques réalisées chez l'animal et exceptionnellement chez l'homme, lors de troubles neurologiques ou d'interventions neurochirurgicales à visée thérapeutique.

Les expériences d'ablation suggèrent que l'aire préoptique médiane (la partie frontale de l'hypothalamus) joue un rôle clé dans le comportement mâle; sa destruction provoque en effet un arrêt plus ou moins total de l'activité copulatoire. Ce blocage est indépendant de l'intensité du désir sexuel : les singes opérés se masturbent ou présentent un comportement femelle lorsqu'ils reçoivent un traitement par des oestrogènes.
A l'opposé, comme on pouvait s'y attendre, la stimulation électrique de la même région chez le mâle provoque une activité copulatrice intense avec une femelle pour peu que celle-ci soit en chaleur, c'est-à-dire désirable. On en conclut que la stimulation de l'aire préoptique diminue le seuil d'activation du comportement sexuel, mais qu'il ne change pas la nature de l'objet de désir.

Nous savons peu de choses sur les connexions entre l'aire préoptique médiane et les autres régions du cerveau. Il existe des sorties sur le noyau dorsomédian de l'hypothalamus, connu pour son rôle dans l'éjaculation, sur les régions motrices qui commandent la gestuelle du coït, et sur les centres végétatifs impliqués dans l'érection.

Chez les mâles castrés, la réduction du désir sexuel et le blocage du comportement copulatoire s'accompagnent d'une diminution de l'activité électrique de l'aire préoptique médiane. L'implantation dans cette même région d'une infime quantité de testostérone restaure la fonction disparue. D'une manière générale, on peut considérer que la testostérone abaisse le seuil d'activation de la région sans modifier la nature des stimuli.

La recherche animale ignore tout des "sentiments" d'une bête incapable d'en parler. Les seules observations humaines dont on dispose ont été obtenues chez des délinquants sexuels ayant subi par neurochirurgie une destruction ménagée de leurs noyaux préoptique et ventromédian de l'hypothalamus. Ces malheureux affirmaient ne plus ressentir de désir sexuel, ni de goût pour ce qui faisait jadis l'objet de leur perversion. Un résultat comparable peut être actuellement obtenu grâce à un traitement médicamenteux, qualifié parfois de castration chimique, qui bloque l'action des androgènes dans le cerveau.

$J$ 'insisterai, pour terminer cette visite du cerveau, sur le rôle de l'amygdale qui constitue, je le rappelle, la clé de voûte du 
cerveau limbique, lequel est spécialisé dans la gestion de nos émotions. Selon la place des électrodes introduites chez des patients épileptiques en vue de détruire la cause des crises que l'on ne pouvait guérir autrement, la stimulation de l'amygdale provoquait une érection ou son inhibition. Une destruction localisée de cette région chez des sujets qui présentaient un comportement agressif criminel n'entraînait pas de modification apparente de leur sexualité. En revanche, l'observation récente d'un patient souffrant d'une maladie d'Urbach-Wiethe, qui avait occasionné chez lui la destruction bilatérale et complète des amygdales, montrait l'incapacité de ce malade d'identifier une émotion d'après son expression faciale. Le sujet ne reconnaissait plus une mimique de peur et ne pouvait plus la différencier de l'expression des autres émotions. Le rôle des amygdales dans la lecture des sentiments sur le visage de l'Autre, place donc cette structure au coeur de la relation amoureuse.

\section{La sexualité masculine et la chaîne érotique}

\section{A. GREEN \\ 9, Avenue de l'Observatoire, 75006 Paris}

On peut envisager la sexualité masculine selon divers niveaux, comme l'indique le programme de cette journée, depuis les structures cérébrales considérées sous l'angle neuro-anatomique ou bio-chimique jusqu'à l'accomplissement de l'acte sexuel amoureux. Mais même lorsqu'on tente de ressaisir l'ensemble de la fonction avec ces différents niveaux de déterminisme, le point de vue psychanalytique institue une rupture qui n'est pas seulement due à l'angle psychique auquel elle s'attaque mais à la manière dont le psychanalyste l'envisa- ge. Sa perspective ne se réclame pas d'une psychobiologie, bien que nous verrons que celle-ci puisse mériter ce nom à un certain titre, ni non plus d'une neuro-endocrinologie qui pourtant pourrait avoir sa part dans les attitudes psychiques des sujets dont nous parlons. Il se pourrait en fait que le point de rupture entre la psychanalyse et les autres disciplines se situe au niveau du refus de considérer le comportement comme l'angle approprié pour l'étude du psychisme. Car cet angle voue l'étude à une évaluation objectivable de l'extérieur de ce qui est constatable par les attitudes extérieures adoptées par les sujets étudiés. Autrement dit, que ni l'intrapsychique ni l'intersubjectif n'y trouvent leur compte. Cette observation vaut également pour distinguer l'approche du sexologue et celle du psychanalyste. En vérité, ce dont s'occupe le psychanalyste est la psychosexualité. Que celle-ci doive ou puisse faire sa place à la psychobiologie au sein de sa théorisation n'empêche pas que cette place soit située au niveau des effets de base qui influent sur l'intégration de la sexualité dans le psychisme mais que c'est le destin des superstructures qui est surtout à prendre en considération. A cet égard, désignée de cette manière, l'infrastructure ne signifie pas qu'en fin de compte ce sera à elle qu'il faudrait revenir. Si c'était le cas, cela ruinerait toute idée de l'efficacité d'un quelconque traitement qui ne serait pas de nature biologique. En d'autres termes, ce dont nous aurons à nous occuper concernera en premier lieu les spécificités de la sexualité humaine et les singularités propres à celleci. Qui ne souhaiterait pouvoir disposer d'un système d'articulations cohérentes entre les différents niveaux d'intervention (chromosomiques, gonadiques, hormonaux,comportementaux, psychiques) qui rende compte des troubles de la fonction sexuelle? Malheureusement, la réalité nous oblige à renoncer pour le moment à cette vision idéale. Outre les cas de lésions grossières entraînant des perturbations 
définitives, il y a le plus souvent manque de concordance entre les divers plans. Des critères biologiques entièrement normaux, au moins dans 1 ' état actuel des connaissances, peuvent coexister avec des perturbations importantes, non seulement du comportement sexuel mais de l'identité sexuelle et du sentiment d'appartenance au sexe biologique (transvestisme, transsexualisme). Inversement, des perturbations d'ordre biologique qui devraient entraîner un désordre parallèle de la sexualité ne voient pas toujours cette opinion confirmée. On voit donc que tout cela plaide en faveur d'une poursuite des recherches. Encore faut-il que celles-ci puissent explorer toute l'étendue du champ à investiguer.

On sait que la sexualité a été placée à l'origine par Freud comme un facteur étiologique à l'origine des névroses. Etrangement, les patients qui souffraient de troubles spécifiques de la fonction sexuelle n'ont pas fait l'objet d'une attention particulière de sa part. Freud a néanmoins étendu l'influence du sexuel très au-delà de sa sphère traditionnelle d'action. Ce n'était pas seulement la névrose qui pouvait bénéficier de cet éclairage mais aussi bien les autres catégories de la nosographie (psychose, dépression etc.) pour peu que l'on tînt compte d'autres facteurs ou encore des transformations de la libido (narcissisme, destructivité, etc.). En fait, cette influence ne s'est pas limitée pour lui à essayer de comprendre la genèse des pathologies. Freud a voulu considérer la sexualité comme un des socles sur lesquels s'édifiaient le psychisme normal et ses déviations, qu'elles soient de l'ordre des fixations ou des régressions. A cette vue, il faut ajouter des précisions. La première concerne l'hypothèse d'une sexualité présente dès le début, idée tout à fait originale à 1'époque, sous-tendant non seulement l'existence d'une sexualité infantile alors inconnue mais aussi celle d'une sexualité présente dès l'origine sous des formes non reconnues comme telles (orales, anales etc.). Cette position de décentrage de la sexualité peut se comprendre par le mouvement, propre à la sexualité humaine proprement dite, de dissociation entre plaisir et reproduction, dissociation qui s'est encore plus accentuée depuis la découverte des techniques de procréation assistée ou au contraire, de contraception. Faire donc remonter le plaisir de zones érogènes. intervenant dans la sexualité accomplie, bien avant toute forme de sexualité génitale était donc tout à fait 1égitime. Ce sont bien les vicissitudes de cette sexualité infantile inaugurale, les refoulements auxquels elle donne lieu, les fixations qu'elle laisse derrière elle, les régressions que son parcours évolutif peut rencontrer, qui constituent un modèle utile pour juger des perturbations de la vie sexuelle des adultes.

En deuxième lieu, ce qu'il convient de ne jamais oublier est que cette accentuation sur le sexuel et sur son rôle déterminant dans la vie psychique ne peut être envisagée isolément. C'est au sein d'une théorie du conflit que s'exprimera la solution choisie souvent sous la forme d'un compromis entre les diverses tendances en antagonisme, Freud a hésité pendant longtemps à définir l'autre terme du conflit, n'ayant jamais cessé par ailleurs d'être convaincu de la constance et de la puissance du facteur sexuel. S'il a fini par opter pour la thèse des pulsions de destruction, surtout sous la forme très controversée des pulsions de mort, il a eu du mal à convaincre une bonne part de ses collègues. La théorie des pulsions de mort est l'objet, jusqu'à aujourd'hui. d'un rejet important de la part des psychanalystes. Sans doute mérite-t-elle d'être reformulée plus qu'écartée. Quoi qu'il en soit, il est peu d'entre eux, à la lumière de I'expérience de la clinique contemporaine, qui a ajouté à la connaissance des névroses celle qu'a révélée l'analyse des états au voisinage de la psychose et, plus généralement, des structures non névrotiques, qui contestent que la destructivité joue un rôle majeur dans son rapport 
conjoint ou antagoniste à la sexualité. Les toutes dernières formulations sur le sexuel, aboutissant de l'oeuvre de Robert Stoller qui y a consacré sa vie, concluent qu'il est dans la nature même de l'excitation sexuelle, expression par ailleurs de l'amour, d'être porteuse d'une teinte, parfois discrète, parfois plus nette, d'hostilite ou d'un désir de nuire, qui témoigne de son alliage avec l'agressivité.

Reste enfin la question du centrage de la sexualite du point de vue du psychisme. Pour Freud, I'utilisation du concept de pulsion - concept-limite disait-il, c'est-à-dire à la fois concept de ce qui est à la limite entre le somatique et le psychique, et peut-être aussi à la limite du conceptualisable - permettait de maintenir l'ancrage de la psychosexualité dans le corps. Par la suite, les auteurs post-freudiens, tentant de dépasser ces hypothèses originaires en rejetant le soi-disant biologisme de Freud, ont cherché d'autres explications en situant ce centre ou ce point d'origine, qui du côté du fantasme, qui du côté du signifiant (énigmatique ou pas), et qui encore du côté du Moi, de ses défenses ou de son adaptivité. Toutes ces conceptions me semblent fautives en ce qu'elles s'égarent a chercher un centre 1à où il vaut mieux penser en termes de chaînes ou de réseaux. J'ai proposé de considérer I'existence d'une chaîne érotique qui partirait de la pulsion et comprendrait, comme élaboration de cette structure où domine la perspective dynamique et sa décharge dans l'acte, la recherche des états de plaisir (avec leur complément obligé, le déplaisir), l'incitation au désir, qui traduit une attitude d'attente et d'espoir alimentée par les représentations inconscientes et conscientes, des fantasmes organisant des scénarios de réalisation de désir et, enfin, l'imaginaire écrit ou figuratif, produit de sublimations plus ou moins poussées, destiné à alimenter la fantasmatique et à soutenir le désir. Ainsi, on disposerait d'une chaîne dont tous les maillons ne sont pas nécessairement présents à tout instant et qui peut être parcourue selon son point de départ, formée par la "mise à feu" de n'importe lequel de ses maillons dans la voie des directions les plus ouvertes, antérogrades ou rétrogrades, selon les censures et les défenses et selon la force de l'excitation sexuelle et son pouvoir d'investissement. Il me semble qu'une telle conception a l'avantage de rendre justice à la complexité du psychisme humain et aussi à sa diversité, comme aux possibilités auxquelles permettent d'assister certains courts-circuits. L'existence de ce qu'on pourrait appeler les "sexualités extrêmes", (sexualité criminelle, délinquance) montre comment ce schéma peut être déformé ou distordu. Par exemple, la référence au plaisir, contrairement à ce qu'on attendrait, peut y disparaître devant un sentiment d'urgence qui relèverait plutôt des impératifs du besoin en conformité avec une activité pulsionnelle cherchant impérativement la décharge dans l'urgence. La contingence de l'objet peut aussi ici prendre toute la place, accordant la priorité absolue à la satisfaction de la pulsion. Quoi qu'il en soit, le travail du psychanalyste, qui pourrait ici s'étendre très au-delà des conditions de possibilité de la cure analytique, dans des conditions permises par l'aménagement du cadre, devrait s'efforcer de reconstituer ou de constituer, aussi complètement que possible, l'ensemble de la chaîne érotique, ce que l'expérience analytique, limitée par des indications précises et par un cadre approprié et adapté, susceptible en certains cas d'assoupplissement, permet de réaliser avec un succès raisonnable, grâce à une technique qui s'est progressivement construite en vue de répondre aux problèmes que posaient les formes de la psychosexualité qui pouvaient y être abordées. 